\title{
Optical Fibers and Their Applications 2014
}

\author{
Jan Dorosz ${ }^{1}$ and Ryszard S.Romaniuk, ${ }^{* 2}$ \\ ${ }^{1}$ Bialystok University of Technology, ${ }^{2}$ Warsaw University of Technology
}

Received May 14, 2014; accepted June 28, 2014; published June 30, 2014

\begin{abstract}
Conference Series on Optical Fibers and Their Applications held its $15^{\text {th }}$ edition in Białystok and Lipowy Most at the end of January 2014. The paper is a concise digest of work results presented there from the main domestic laboratories active in optical fiber and planar photonics. The conference topics were: optical fiber technology, materials for optoelectronics and photonics, rare earth doped and luminescent materials, metrology of optical fibers, components and optoelectronic circuits, applications of optical fibers, waveguides and optical fiber sensors, and lighting technology. A ful version of the OFTA2014 digest paper was published in [1]. The proceedings of previous OFTA conferences were published in [2-3].
\end{abstract}

Technological work on optical fibers is pursued in Poland in three laboratories: UMCS Lublin, Białystok University of Technology (BUT), and ITME Warsaw. Optical fibers manufactured in these laboratories are applied for material and technological research of fiber pulling processes, and to build functional photonic components. The production of telecommunication type optical fibers, photonic optical fibers, and non-telecom specialty fibers in these laboratories, is beneficial to numerous electronic, metrological, telecommunications and photonic, university based laboratories, in this country and abroad. Optical fibers were used for building of components, circuits, devices and photonic systems. Due to the availability of high quality optical fibers, of domestic production, telecom and non-telecom ones, there were developed numerous, modern, university based laboratories specializing in optical fiber photonics. This is a considerable, yet not overestimated, success of these national technological laboratories, among which the Laboratory of Optical Fiber Technology at UMCS in Lublin, chaired at that time by prof. A. Waksmundzki, was the first one. Soon, the optical fiber technology community in Poland will celebrate its fortieth anniversary.

Optical fiber and waveguide photonics is a hot subject which involves numerous optical laboratories in Poland and elsewhere. The following examples presented during the OFTA2014 conference show general development directions of this field. Some of these examples are related to potentially useful applications, which are not possible to be achieved using only conventional optical fibers:

\footnotetext{
*E-mail: r.romaniuk@ise.pw.edu.pl
}

- Transmission of a low-loss and stable, linear (without destroying the fiber) strictly single-mode beam in the UV range (spectral range 200-300nm) - for use in highly integrated electronics, to manipulate trapped ions, to stimulate biological molecules, to do micro-marking, etc. ;

- Ultra-low-loss transmission of waves in the whole area of the telecommunications spectrum $(800-1700 \mathrm{~nm})$ in the photonic fiber capillary - to construct low latency links, at present the best solutions of low-loss optical fibers have $1.5 \mathrm{~dB} / \mathrm{km}$ at $1600 \mathrm{~nm}$;

- Ultra-broadband VIS, IR, and MIR transmission using ultra- purified soft glass, ZBLAN glasses and fibers - to build super-continuum light sources; usage of strong nonlinear functional transformation of the optical signal to transmit the high power IR wave, and for spectroscopic applications;

- Transmission of a wavelength in the range of MIR and FIR, particularly in the area of $\sim 10 \mu \mathrm{m}$, for the purpose of molecular chemistry, biochemistry, biophotonics, spectroscopy, neurophotonics, genomic-photonics, and $\mathrm{THz}$ technology;

- Construction of components, circuits and systems of organic photonics with extensive use of lasers and detectors, polymeric photonics of new generation, polymer fibers including active ones.

Organic Photonics, which is currently under intensive development, evokes hopes to build full functional systems performed in a single technology. The basis is polymers, of which are built discrete light sources, OLED panels, detectors, waveguides, planar photonic functional components - discrete and organized in matrices, optical fibers for transmission, active as well as classical, and photonic fibers. The doping of low-loss polymers with optically active ions is associated with avoiding the luminescence suppression effect of the high-energy phonons in polymers. Work is continuing in the Warsaw University of Technology (WUT), IMiO on intermediate doping of polymers for the purpose of a light source and fibers, using active ion doped in inorganic nanocrystallites. Technological problems encountered during these hybrid processes are: hygroscopic nature of nano-powders, increased optical loss of the polymer, the challenged mechanical properties of nano-powders doped crystalline polymer, etc. Indirect doping avoids a direct 
effect on the fluorescence quenching of the polymer matrix.

Polymer optical fibers are manufactured in national laboratories of fiber optic technology in conventional multimode versions, singlemode, and photonic, solid and hollow. They are produced by drawing or extrusion methods. The technological issue is to minimize optical loss and the stabilization of geometric dimensions, etc. The theoretical work, carried out at UMCS, concerns combined parameters of processes occurring in fiber drawing and, in particular, a precise analysis of the gaseous product of the reaction, mass change of the sample, as well as the composition of the material, temperature and speed of the process. One group of materials used to make optical fibers is poli-cycloolephines, which are polymers of good optical and mechanical properties, and high water resistance. The glass transition temperature is above $130^{\circ} \mathrm{C}$, and fiber production technology is stable. Polymer optical fibers with PMMA and Zeonex (cyclo-olephine) filled with nematic liquid crystals (WUT, Faculty of Physics) are examined against the changes in the fiber transmission as a function of temperature. PMMA was repeatedly doped with organic dyes such as fluoresceine and rhodamine, for testing resonance energy transfer in the polymer - FRET (PB). The aim is to develop materials for polymer active fibers. Optical fibers doped with optically active polymer nano-composites are candidates for a new generation of light sources. Work on such solutions is carried out at WUT, IMiO in collaboration with UMCS. Organicmetallic complexes doped with dysprosium show luminescence in the visible band, as well as inorganic doped lanthanides. Luminescent nano-polymer composites, as well as three-dimensional coordination polymers of rare earth elements are examined in order to build active optical fibers and light sources in the visible and telecommunication bands.

The impact of the plasmonic phenomena must be felt in the core area of a fiber optic sensor. The location of the plasmonic electrode has to be within a nanometer distance from the evanescent field of guided mode carried in the core of the optical fiber or planar optical waveguide, single mode or multimode. Fiber-optic plasmonic sensors made of glass and polymer are studied in many laboratories (WUT, Wrocław Univ. of Technology WrUT, Silesian Univ. of Technology, and others). The technological problems are: the homogeneity of the metal layer, dimensions of individual elements of the sensor, method of application and polishing and/or etching the layers, method of preparation of the fiber to the imposition of the sensor, etc. The measure of quality is the position of plasmonic resonance and its width at half maximum. Plasmonic sensors have been used as strain gauges, refractometers, etc.

The light sources used in fiber optic technology are subject to evolution towards increasing radiated power, miniaturization, ease of powering, wavelength stability, thermal stability, possibility of controlled and precise tuning, ease of coupling with fiber, wavelength generation in other spectral bands than telecommunications, such as UV, MIR, THz, etc. A separate group of sources of radiation, which is subject to particularly intensive development,is fiber supercontinuum sources. In the classical solution, they have known limitations in the form of uneven and unstable spectral characteristics. Ongoing works concern the characteristics equalization and its stability, extend the spectral range of generated spectrum, the spectrum shifts toward the MIR and THz, etc. The works are carried out in WrUT but also in other laboratories in Poland. Supercontinuum is generated in conventional optical fibers (where the nonlinear effects are small) and in specialized optical fibers of soft glass, full-core, photonic and capillary ones. The classic solution uses anomalous dispersion fiber for supercontinuum sources. New solutions use fibers from a variety of materials with normal dispersion across the whole transmittance band pumped with ultra short pulses. Such an ultra-wideband fiber optic light source was used, with considerable success, in the ultra-sensitive gas spectroscopes operating in the IR band for measurements in medical, environmental and industrial applications (WAT).

Fiber optic, photonic, broadband, high-power light sources of the supercontinuum type displace other sources used in spectroscopic systems and in a variety of other applications. Photonic fiber capillary gives the designer considerable freedom to shape the detailed properties of such sources. Capillary optical fibers give a photonic source parameters which can not be obtained in conventional optical fibers. The zero dispersion wavelength in capillary photonic fiber (the second derivative of eigen-refraction) can be moved technologically within any range of transparent fibers spectrum, e.g. from 380 to $2400 \mathrm{~nm}$. The fiber can be unconditionally single mode throughout this spectral range. The optical pump needs to be adjusted to the zero dispersion wavelength, for effective generation of the supercontinuum. Mutual adjustment of many technology parameters of the photonic hollow fiber, like bandwidth transparency, zero dispersion, luminescent characteristics (in the active fiber), optical pump, shape of the fibers (conical or multi-conical) leads to optimal solutions for new light sources, radiating uniformly throughout the transparency range of the fiber. Technological parameters are adjustable, e.g. capillary diameter, core diameters, 
form of nano-capillary cladding, structure of the border areas between the capillaries, capillary layout, refraction of glass or polymer, etc. Capillary fiber is a high dispersion device and can be optically linear or highly non-linear - the last parameter depending on the material. Work on such highly optimized fiber optic light sources, with flat spectral characteristics of radiation, is carried out in some centers in Poland (Military Univ. of Technology, UMCS, ITME, WrUT, WUT and others). During the production of photonic fibers for supercontinuum sources, and for other uses, it is important to remember that the technological parameters have tolerances. The processes of formation of the photonic fibers are very sensitive to changes in technological parameters, which leads to considerable changes of signal parameters of the resulting application oriented fiber.

Fiber supercontinuum source using silica optical fibers is to operate only at wavelength till around $2400 \mathrm{~nm}$. Construction of a stable source of classic ZBLAN fiber allows optimum broadband light generation in the spectral range of 1.5-4.5 microns, and at the same photonic capillary optical fiber the band extends to 5 microns. The use of chalcogenide fibers allows one to move the boundary of about 9 microns. From the point of view of potential applications in molecular chemistry and $\mathrm{THz}$ technology, in medicine for detection of cancer, in the food industry to determine the quality of products, etc., it is important to extend the generation of the band area above 10 microns. The test materials are researched for such photonic fibers at ITME, BUT, and UMCS.

The distribution of precision standard time and frequency is performed in specially constructed fiber optic networks. Work on the fiber distribution of the atomic clock (TAI, UTC - international time standards) is conducted at AGH-University in Kraków, and WUT in collaboration with CERN and DESY. In such distribution systems of precise time, the transmission return channel is required to be synchronized. Propagation delay is actively stabilized by electronic means. This ultra-precision fiber optic network connects some of the atomic clocks located at different places in Poland. A high precision fiber optic network synchronization system, by extended PTP standard - White Rabbit, is developed to synchronize large research experiments.

A consortium of science and technology under the guidance of AGH (GUM-Central office of Measures [4], CBK-Space Research Centre [5], Orange, PCSS -Poznań Supercomputing and Networking Center) implements the project OPTIME to build a national fiber optic distribution network of time and frequency. There was built a 420-kilometer fiber optic link between the GUM in Warsaw and laboratory CBK in Borowiec. There have been performed comparisons of long-term time scales
UTC-PL and UTC-CBK with the stabilized fiber link. In addition, these scales were compared to time scales using GPS methods. It is planned to branch the fiber optic network of atomic clocks to university laboratories in Torun and the astronomical observatory in Piwnice near Torun. It is planned to connect the national time reference network to the European network.

Next generations of ultra-broadband fiber networks require more detailed analysis and supervision of individual communication channels. Supervision of lines with speeds over 40Gbps requires knowledge of polarization mode dispersion PMD of the first order, ie, the differential group delay DGD, and the division of power transmitted in two orthogonal polarization modes. The PMD parameter can be determined from the shape of the transmitted signal, i.e. the input and comparison to the signal received. The measurement allows to compensate distortion by changing the dispersion shape of the transmitted signal. Work on the PMD parameter estimators of the first and higher orders is carried out at the TU Lublin. The use of estimators allows for better management of the transmission channels of very high data rates.

Work on the elements for advanced fiber-optic transmission systems including: cyclical sources for UDWDM, commutators with the conversion of optical waves and other similar devices is carried out at the PUT.

OFTA 2014 gathered relevant representatives of major optical fiber laboratories in Poland. The conference adequately showed the development of this field. The next $\mathrm{XVI}^{\text {th }}$ conference on Optical Fibers and Their Applications will be organized in autumn 2015 in Lublin and Nałęczów. The co-organizers are the Lublin University of Technology and UMCS, Laboratory of Optical Fiber Technology. The conference will celebrate $40^{\text {th }}$ anniversary of establishment of Optical Fiber Technology at UMCS University in Lublin.

\section{References}

[1] R.S. Romaniuk, J. Dorosz, (Edit.) Optical Fibers and Their Applications 2014, Proc. SPIE 9228 (2014).

[2] J. Dorosz, R.S. Romaniuk (Edit.), Optical Fibers and Their Applications 2011, Proc. SPIE 8010 (2011), doi:10.1117/12.901553

[3] R.S. Romaniuk, W. Wójcik (Edit.), Optical Fibers and Their Applications 2012, Proc. SPIE 8698 (2012), doi:10.1117/12.2020581

[4] Central Office of Measures http://www.gum.gov.en

[5] Space Research Centre, Polish Academy of Sciences http://www.cbk.waw.p1/en/ 